\title{
ON QUOTIENT VARIETIES AND THE AFFINE EMBEDDING OF CERTAIN HOMOGENEOUS SPACES $\left({ }^{1}\right)$

\author{
1 BX \\ MAXWELL ROSENLICHT
}

We recall that if an algebraic group $G$ operates regularly on a variety $V$, by a quotient variety is meant a pair $(V / G, \tau)$, where $V / G$ is a variety and $\tau: V \rightarrow V / G$ is a rational map, everywhere defined and surjective, such that two points of $V$ have the same image under $\tau$ if and only if they have the same orbit on $V$, and such that, for any $x \in V$, any rational function on $V$ that is $G$-invariant (i.e., constant on orbits) and defined at $x$ is actually (under the natural injection of function fields $\Omega(V / G) \rightarrow \Omega(V), \Omega$ denoting the universal domain) a rational function on $V / G$ that is defined at $\tau x$ (cf. [1, exposé 8]). $\Omega(V / G)$ must therefore consist precisely of all $G$-invariant elements of $\Omega(V)$, so $\tau$ is separable. A quotient variety need not exist (obvious necessary condition: all orbits on $V$ must be closed), but when it exists it is clearly unique to within an isomorphism; in this case, for any open subset $U \subset V / G, \tau^{-1} U / G$ exists and equals $U$.

PROPOSITION 1. Let the algebraic group $G$ operate regularly on the variety $V$, all defined over the field $k$. Suppose there exists a quotient variety $\tau: V \rightarrow V / G$. Suppose also that for each point $p$ of $V$ that is algebraic over $k$ there exists an open affine subset of $V / G$ containing the image under $\tau$ of each of the conjugates of $p$ over $k$ (a vacuous condition if. $V / G$ can be embedded in a projective space or if $V / G$ and $\tau$ are known to be defined over a regular extension of $k$, in particular if $k$ is algebraically closed). Then $V / G$ and $\tau$ could have been taken so as to be defined over $k$.

The $G$-invariant elements of $\Omega(V)$ are generated by those in $k(V)$, in other words there exists a variety $W$ and a generically surjective rational map $V \rightarrow W$, both defined over $k$, such that for any field $K$ between $k$ and $\Omega$, $K(W)$ is the field of $G$-invariant elements of $K(V)$ [3, Theorem 2]. We have here a field descent problem, and supposing $V / G$ and $\tau$ to be defined over the extension field $K$ of $k$, there are two cases to consider: $K$ a regular extension of $k$, and $K$ algebraic over $k$. In view of the unicity to within isomorphism of the quotient variety; the criteria of Weil [6] take care of the first case. [Of course this can also be done directly; e.g., supposing $k$ algebraically closed, if

Received by the editors February 28, 1961.

(1) This research was supported by the United States Air Force through the Air Force Office of Scientific Research of the Air Research and Development Command under Contract AF 49(638)-603. 
$K=k(t)$, where $(t)$ is a generic point over $k$ of some variety defined over $k$, and if the $G$-invariant elements of $k(V)$ are $k\left(x_{1}, \cdots, x_{r}\right)$, for any $p \in V$ that is rational over $k$ there are coordinate functions valid in some neighborhood of $\tau p$ in $V / G$, say $\phi_{1}(x, t), \cdots, \phi_{n}(x, t) \in k(x, t)$, and these will be such under a suitable $k$-specialization of $(t)$ into a point rational over $k$, and we have then only to worry about enough points $p$.] In the second case, $K$ algebraic over $k$, Weil's results may not apply since the extension need not be separable, so we use another argument: For any point $p \in V$ that is algebraic over $k$ let $U \subset V / G$ be an open affine subset containing the image under $\tau$ of each of the conjugates of $p$ over $k$. Suppose $U$ to be $K^{\prime}$-open, where $K \subset K^{\prime} \subset \Omega$. The coordinate functions on $U$ are then in $K^{\prime}(V / G)$, but a suitable $K$-specialization into the algebraic closure of $K$ will give a set of functions on $V / G$ that are defined over an algebraic extension of $K$ and are local coordinate functions for $V / G$ in a neighborhood of the finite set of points in question; cutting this last neighborhood down suitably shows we could have assumed $K^{\prime}$ to be algebraic over $K$. Changing $K$ if necessary, we assume that $U$ is $K$-open, with $K$ a finite normal algebraic extension of $k$. For any $k$-automorphism $\sigma$ of $K, \tau^{\sigma}: V \rightarrow(V / G)^{\sigma}$ is also a quotient variety, hence isomorphic (over $K$ ) to $\tau: V \rightarrow V / G$. The subset $U_{\sigma} \subset V / G$ corresponding to $U^{\circ} C(V / G)^{\circ}$ under our birational equivalence is also $K$-open and affine and contains our finite set of points. Replacing $U$ by the affine set $\Pi_{\sigma} U_{\sigma}$ we get $\left(\tau^{-1} U\right)^{\circ}=\tau^{-1} U$, i.e., $\tau^{-1} U$ is $k$-open. Since such $k$-open subsets cover $V$, it suffices to prove the proposition for the case $V / G$ affine. But this is easy: any coordinate function on $V / G$ is an everywhere defined function on $V$, hence of the form $\sum c_{i} f_{i}$, with the $c_{i}$ 's in $\Omega$ and linearly independent over $k$, and the $f_{i}^{\prime}$ 's everywhere defined functions in $k(V)$ (cf. the result stated below in the proof of Lemma 1 of Theorem 1). Linear disjointness implies that each $f_{i}$ is $G$-invariant, hence everywhere defined on $V / G$. Enough such functions $f_{i}$ give an affine representation of $V / G$ over $k$.

The following extremely useful result is due to Chevalley [2, pp. 195196]. For the convenience of the reader, we outline a proof.

Lemma 1. Let $V, W$ be varieties, let $\tau: V \rightarrow W$ be an everywhere defined generically surjective rational map, and let $p \in V$ be such that $\tau p$ is normal on $W$ and each component of $\tau^{-1}(\tau p)$ that passes through $p$ has the correct dimension $\operatorname{dim} V-\operatorname{dim} W$. Then the image under $\tau$ of any neighborhood of $p$ is a neighborhood of $\tau p$.

Let $x_{1}, \cdots, x_{a}, a=\operatorname{dim} V-\operatorname{dim} W$, be functions on $V$ that are defined at $p$ and such that $p$ is a component of $\tau^{-1}(\tau p) \cap\left(x_{1}=\cdots=x_{a}=0\right)$; replacing $W$ by $W \times \Omega^{a}$ and $\tau$ by the map $P \rightarrow\left(\tau P, x_{1}(P), \cdots, x_{a}(P)\right)$, we are reduced to the case $\operatorname{dim} V=\operatorname{dim} W$. This being so, we may alter first $W$, then $V$, so as to be able to assume $W$ projective and normal. Let the abstract field $K$ be a normal algebraic extension field of $\Omega(W)$ containing $\Omega(V)$, and let $V^{\prime}$ be 
the normalization of $W$ in $K$. If $p^{\prime} \in V^{\prime}$ is a point corresponding to $p$, then $p$ is an isolated point of the subset of $V$ that corresponds to $p^{\prime}$, so the rational map $V^{\prime} \rightarrow V$ is defined at $p^{\prime}$. Replacing $V$ by $V^{\prime}$, we are reduced to the case where $W$ is complete and normal and $V$ is the normalization of $W$ in a finite normal algebraic extension field of $\Omega(W)$. Now each $\Omega(W)$-automorphism $\sigma$ of $\Omega(V)$ induces an automorphism of the covering $V \rightarrow W$, and the various $\sigma$ 's permute transitively the points of $V$ lying over any given point of $W$ (to verify the latter statement, note that we may consider the analogous statement when $W$ is affine; then $V$ is also affine, and for every $\zeta \in \Omega[V], \Omega[V]$ being the ring of everywhere defined functions on $V$, we have a $\dot{p}^{\prime}$ th power of $\prod_{\sigma}\left(Z-\zeta^{\sigma}\right)$ contained in $\Omega[W][Z]$, so the various values of $\zeta$ at the points of $V$ lying over any given point of $W$ are determined). Thus we must show that the image under $\tau$ of any open subset of $V$ that is invariant under each $\sigma$ is an open subset of $W$, and this is a consequence of the fact that a proper image of a closed set is closed. Q.E.D.

LEMMA 2. Let the algebraic group $G$ operate regularly on the variety $V$ and let $\tau: V \rightarrow W$ be an everywhere defined surjective rational map such that two points of $V$ have the same image under $\tau$ if and only if they have the same orbit. Then all orbits on $V$ have the same dimension. If $W=V / G$ (a case that will certainly hold if $\tau$ is separable and $W$ normal), then the map $\tau$ is open; furthermore if we denote normalization by *, and by $\tau^{*}$ the rational map $V^{*} \rightarrow(V / G)^{*}$ corresponding to $\tau$, then $\tau^{*}$ is everywhere defined and, for any $p \in V, \tau^{*}$ maps the points of $V^{*}$ lying over $p$ onto the points of $(V / G) *$ lying over $\tau p$.

For any transformation space $V$ for $G$, let $\Phi \subset V \times V$ be the locus of ( $v, g v), v \in V, g \in G$, and $\Gamma \subset G \times \Phi \subset G \times V \times V$ be the graph of the operation of $G$ on $V$. Then the isotropy group of any $p \in V$ is given by $\Gamma \cap(G \times p \times p)$, hence has dimension $\geqq \operatorname{dim} \Gamma-\operatorname{dim} \Phi$, which is the dimension of the isotropy group of a generic point of $V$. Thus the dimension of any orbit is majorized by that of the generic orbit. On the other hand, since we have our map $\tau$, the dimension of any orbit is at least that of the generic orbit; thus all orbits have the same dimension. If $W$ is normal, the openness of $\tau$ follows from Lemma 1 ; also if $p \in V$ and $f \in \Omega(W)$ is defined at $p$, then $f$ must be defined at $\tau p$, for the image under $\tau$ of the domain of definition of $f$ on $V$, an open subset of $V$, contains a neighborhood of $\tau p$, so the polar locus of $f$ on $W$ can have no component through $\tau p$, the function $1 / f$ on $W$ being defined along any such component and taking the value 0 . If $\tau$ is separable, clearly any $G$-invariant function on $V$ is in $\Omega(W)$, so $\tau$ separable and $W$ normal implies $W=V / G$. Now drop the assumption that $W$ is normal, but suppose $W=V / G$. Each point of $V^{*}$ corresponds under $\tau$ to a definite point of $V / G$, hence to one of a finite number of points of $(V / G)^{*}$. By Zariski's Main Theorem, $\tau^{*}$ is everywhere defined. Trivially, for any $p \in V, \tau^{*}$ maps the points over $p$ into points of $(V / G) *$ lying over $\tau p$. Suppose now that $q$ is a point of $(V / G) *$ lying 
over $\tau p$ that corresponds to no point of $V^{*}$ lying over $p$. Then there exists a function $f \in \Omega(V / G)$ that is not defined at $q$ but is defined at all other points of $(V / G)^{*}$ lying over $\tau p . f$ is then defined at all points of $V^{*}$ lying over $p$, hence is integral over the local ring $\mathfrak{o}_{p}$. Writing $f=x / y$, with $x, y \in \mathfrak{o}_{r p}$, there is an integer $n$ such that $y^{n} f^{*} \in 0_{p}$ for all $s \geqq 0$. Hence $y^{n} f^{s} \in 0_{r p}$, so $f$ is integral over $o_{r p}$, hence defined at $q$, contrary to assumption. It remains to verify that $\tau$ is open. But any open neighborhood of $p$ corresponds to an open subset of $V^{*}$ containing each point of $V^{*}$ lying over $p$, hence (by Lemma 1) maps under $\tau^{*}$ into an open subset of $(V / G)^{*}$ containing each point of $(V / G)^{*}$ lying over $\tau p$, hence (by the closedness of the mapping into any variety of its normalization) produces a neighborhood of $\tau p$.

Lemma 2 shows that if $V / G$ exists, its topology must be the quotient topology. The lemma also implies that if $V$ is a connected algebraic group and $G$ an algebraic subgroup operating by right translation, then the usual homogeneous space $V / G$ is actually a quotient space in the present sense. We remark that in [1] Chevalley has defined quotient spaces for more general fiberings of a variety than that given by a group operation, and Lemma 2 extends immediately to this more general case, at least under the assumption that all fibers have the same dimension.

LEMma 3. Let the algebraic group $G$ operate regularly on the variety $V$ in such a way that $V / G$ exists, and let $W$ be another variety. If $G$ operates on $V \times W$ by the rule $g(v, w)=(g v, w)$, then $(V \times W) / G$ exists and equals $(V / G) \times W$.

The rational map $V \times W \rightarrow(V / G) \times W$ is everywhere defined, separable, and has the correct set-theoretic properties. We therefore need only show that if $(v, w) \in V \times W$ and $f \in \Omega((V / G) \times W)$ is defined at $(v, w)$, then $f$ is also defined at $(\tau v, w), \tau$ denoting the natural map $V \rightarrow V / G$. Without loss of generality we may replace $W$ by an affine neighborhood of $w$, and similarly $V / G$ may be replaced by some affine neighborhood of $\tau v, V$ being cut down suitably. Thus we may suppose that $V / G$ and $W$ are affine varieties. Letting * denote normalization, there is a natural everywhere defined rational map $V^{*} \times W^{*} \rightarrow(V / G)^{*} \times W^{*}$ and this map is open, by the previous lemmas. The polar divisor $F$ of $f$ on the normal variety $(V / G)^{*} \times W^{*}$ can have no component passing through any point lying above $(\tau v, w)$, for otherwise $1 / f$ would be defined and take the value zero along such a component, hence (by the openness of the above map and Lemma 2) along a subvaricty of $V^{*} \times W^{*}$ passing through a point above $(v, w)$, which is impossible. The image $F^{\prime}$ of $F$ on $(V / G) \times W$ is a closed subset not passing through $(\tau v, w)$, so there is a polynomial function $\phi$ on $(V / G) \times W$ vanishing on $F^{\prime}$ but not at $(\tau v, w)$. For some $\nu>0, \phi^{y} f$ will have no poles on $(V / G)^{*} \times W^{*}$, hence will be everywhere defined, i.e., be a polynomial function on the affine variety $(V / G)^{*} \times W^{*}$. Replacing $f$ by $\phi^{\prime} f$, we may thus suppose that $f$ is of the form $f=\sum_{i=1}^{n} f_{i} h_{i}$, where each $f_{i} \in \Omega(V / G)$ and each $h_{i} \in \Omega(W)$. Suppose $f_{1}, \cdots, f_{n}$ linearly independent 
over $\Omega$, and similarly for $h_{1}, \cdots, h_{n}$. Since $f$ is defined at $(v, w)$ it is defined along $v \times W$; say $f$ is defined on $v \times(W-Z)$, where $Z \neq W$ is a closed subset. Choose $w_{1}, \cdots, w_{n} \in W-Z$ such that each $h_{i}$ is defined at each $w_{j}$ and $\operatorname{det} h_{i}\left(w_{j}\right) \neq 0$. For each $j=1, \cdots, n$, the function $\sum f_{i} h_{i}\left(w_{j}\right)$ on $V$ is defined at $v$, so each $f_{i}$ is defined at $v$. In the same way, each $h_{i}$ is defined at $w$. Since $V / G$ is a quotient variety, each $f_{i}$, considered as a function on $V / G$, is defined at $\tau v$. Hence $f=\sum f_{i} h_{i}$ is defined at $(\tau v, w)$. Q.E.D.

Before proceeding, we mention the following application of Lemma 3 to an often arising question: If the algebraic group $G$ operates regularly on the variety $V=\bigcup_{i \in I} V_{i}$, where each $V_{i}$ is a $G$-invariant open subset such that $V_{i} / G$ exists, then does $V / G$ exist? To answer this, consider the subset $F=\{(v, g v) \mid v \in V, g \in G\} \subset V \times V$, i.e., the set-theoretic projection on $V \times V$ of the graph of the operation of $G$ on $V$. If $V / G$ exists, then $F$ is the inverse image of the diagonal under the obvious rational map $V \times V \rightarrow(V / G) \times(V / G)$, hence closed. Conversely, if $F$ is closed then $V / G$ exists: for let $\tau_{i}: V_{i} \rightarrow V_{i} / G$, $i \in I$, be the natural maps, and set $V / G=\bigcup_{i \in I} V_{i} / G$ with the obvious identifications, i.e., for $v \in V_{i} \cap V_{j}$ we identify $\tau_{i} v$ and $\tau_{j} v$. The only point that needs checking is that for $i, j \in I$, the set $\left\{\left(\tau_{i} v, \tau_{j} v\right) \mid v \in V_{i} \cap V_{j}\right\}$ is a closed subset of $\left(V_{i} / G\right) \times\left(V_{j} / G\right)$. But this set is the image of $F \cap\left(V_{i} \cap V_{j} \times V_{i} \cap V_{j}\right)$, under the map $\left(v_{i}, v_{j}\right) \rightarrow\left(\tau_{i} v_{i}, \tau_{j} v_{j}\right)$, and closure results from the facts that $F \cap\left(V_{i} \cap V_{j} \times V_{i} \cap V_{j}\right)$ is a closed $G \times G$-invariant subset of the variety $V_{i} \times V_{j}$ (on which $G \times G$ operates regularly), that $\left(V_{i} \times V_{j}\right) /(G \times G)=\left(V_{i} / G\right)$ $\times\left(V_{j} / G\right)$ (repeated application of Lemma 3 ), and that a map into a quotient variety is open (Lemma 2). The set $F$ is automatically closed if $G$ is finite (in which case the closure of $F$ follows from the closure of the diagonal of $V \times V$ ) or if $V \times V=U_{i \in I} V_{i} \times V_{i}$, i.e., if any given pair of points of $V$ is contained in at least one $V_{i}$. (This last condition is due to Chevalley $[1$, p. 8-06], and its sufficiency can be shown directly in a very easy way: indeed, returning to the details above, for any $(p, q)$ in the closure of $\left\{\left(\tau_{i} v, \tau_{j} v\right) \mid v \in V_{i} \cap V_{j}\right\}$ there exists $V_{k}$ such that $\tau_{i}^{-1} p, \tau_{j}^{-1} q \subset V_{k}$; the facts that the birational correspondence from $\left(V_{i} / G\right) \times\left(V_{j} / G\right)$ to $\left(V_{k} / G\right) \times\left(V_{k} / G\right)$ is biregular at $(p, q)$ and that the diagonal on $\left(V_{k} / G\right) \times\left(V_{k} / G\right)$ is closed give $\left.\tau_{i}^{-1} p=\tau_{j}^{-1} q\right)$. We note with Chevalley that the last condition is certainly satisfied (at least if the set $\left\{V_{i}\right\}_{i \in I}$ is chosen large enough) if $V$ also happens to be a homogeneous space for another algebraic group $H$, the operations of $G$ and $H$ on $V$ commuting, for then any given point pair on $V$ is contained in a suitably generic translate of any $V_{i}$.

The necessity for proving the following fact, especially in the case where $H$ operates trivially on $V$ (which occurs several times in the literature), seems not to have been noted.

Proposition 2. Let the algebraic group $G$ operate regularly on the variety $V$, and let $H$ be a normal algebraic subgroup of $G$ such that $V / H$ exists. Then the operation of $G / H$ on $V / H$ is regular. 
The latter operation is given by $(\mathrm{gH}, H v) \rightarrow H g v$, at least generically, and we must show that this rational map $(G / H) \times(V / H) \rightarrow V / H$ is everywhere defined. Letting $H \times H$ operate on $G \times V$ by the rule $\left(h_{1}, h_{2}\right)(g, v)=\left(g h_{1}^{-1}, h_{2} v\right)$, the rational map $G \times V \rightarrow V / H$ that is given by $(g, v) \rightarrow H g v$ is everywhere defined and $H \times H$-invariant so, assuming that $(G \times V) /(H \times H)$ exists, the map $(G \times V) /(H \times H) \rightarrow V / H$ is everywhere defined. But a double application of the last lemma shows that $(G \times V) /(H \times H)$ indeed exists and equals $(G / H) \times(V / H)$, so we are done.

Note that, under the conditions of Proposition 2, $(V / H) /(G / H)$ exists if and only if $V / G$ exists, in which case they are equal.

COROLlARY. Let $V$ be a variety and let $G$ be an algebraic group that operates regularly on $V$. Let $G^{\prime}$ be another algebraic group that operates on $V$ and let $\phi: G \rightarrow G^{\prime}$ be a surjective rational homomorphism such that $g(v)=\phi g(v)$ for generic $g \in G, v \in V$. Then $G^{\prime}$ operates regularly on $V$.

Simplify slightly by taking $G$ connected. The proposition enables us to assume that $\phi$ is a purely inseparable isogeny, so that we are in the case of characteristic $p \neq 0$. By the results of Serre [5], we have to show that if $\mathfrak{n}$ is the kernel of the Lie algebra map associated with $\phi$, then $G / \mathfrak{n}$ operates regularly on $V$. To do this we must first consider the following analogy of the preceding part of this paper. Let $V$ be a variety that is defined over a field $k$ of characteristic $p \neq 0$ and let $\mathfrak{N}$ be a $p$-Lie subalgebra of the derivations of $\Omega(V) / \Omega$. By a quotient variety for $V$ over $\mathfrak{N}$ we mean a pair $(V / \mathfrak{N}, \tau)$, where $V / \mathfrak{N}$ is a variety and $\tau: V \rightarrow V / \mathfrak{N}$ a bijective rational map such that if $f \in \Omega(V)$ is defined at a point $x \in V$ then $f$ is a rational function on $V / \Re$ that is defined at $\tau x$ if and only if $f$ is annulled by $\mathfrak{N}$. The unicity of such a quotient is clear, and we must show that one exists, and in fact that if $\mathfrak{N}$ has a basis consisting of derivations of $k(V) / k$ then $V / \mathfrak{N}$ and $\tau$ may be taken to be defined over $k$. To do this, we may suppose $V$ affine, and here we can show that $V / \mathfrak{N}$ is also affine: in fact, we take for the affine ring of $V / \mathfrak{N}$ over $k$ the subring of $k[V]$ that is annulled by $\mathfrak{N}$, a finitely generated ring over $k$ over which $k[V]$ is a finite module, since $k[V]$ is integral over its subring $k\left[(k[V])^{p}\right]$. Next let $V, \mathfrak{N}$ be as above and let $W$ be another variety. Identifying $\mathfrak{N}$ with an algebra of derivations of $\Omega(V \times W) / \Omega$ we have, analogous to Lemma 3, an isomorphism between $(V \times W) / \mathfrak{N}$ and $(V / \mathfrak{N}) \times W$; the proof of this is trivial in the affine case. For our present problem we put $\mathfrak{l}=\mathfrak{n} \otimes_{\Omega} \Omega(V)$, so $G / \mathfrak{N}$ $=G / n$. Now reason as in the proposition: the regular map $G \times V \rightarrow V$ goes through $(G \times V) / \mathfrak{N}=(G / \mathfrak{N}) \times V=(G / \mathfrak{n}) \times V$, and we are done. (It is clear that all this is susceptible of a much wider generalization, which can only be done in the Grothendieck language.)

LEMMA 1. Let the algebraic group $G$ operate regularly on the variety $V$, all defined over the field $k$. Then any everywhere defined rational function on $V$ is 
contained in a finite dimensional vector space $S$ of such functions that is invariant under translation by elements of $G$, and $S$ may be taken to have a basis consisting of functions that are defined over $k$. If such a basis for $S$ is chosen, we obtain a surjective rational homomorphism, defined over $k$, from $G$ into an algebraic group of matrices.

This is a slight generalization of [3, Theorem 12], where $V$ was assumed nonsingular, and can itself be generalized in the obvious manner to the case where $V$ is reducible. The proof is exactly the same as that of the quoted result if we replace the last theorem of Weil's Foundations by the following result: If $V$ is a variety defined over $k$, then any everywhere defined rational function $f$ on $V$ is of the form $f=\sum c_{i} f_{i}$, where each $c_{i} \in \Omega$ and each $f_{i} \in k(V)$; furthermore, if the $c_{i}$ 's are taken to be linearly independent over $k$, then each $f_{i}$ is defined everywhere on $V$. To prove this it suffices to take $V$ affine, in which case the everywhere defined rational functions are precisely the polynomial functions, and these latter are the tensor product of $\Omega$ with the ring of polynomial functions that are defined over $k$.

Define a variety to be quasi-affine if it can be embedded in an affine space, i.e., is isomorphic to an open subset of an affine variety. A (quasi-) affine variety that is defined over $k$ is isomorphic over $k$ to (a $k$-open subset of) an affine variety that is defined over $k$ : this is an easy consequence of the result quoted in the proof of the preceding lemma.

LEMMA 2. If the algebraic group $G$ operates regularly on the quasi-affine variety $V$, everything being defined over $k$, then there exists an embedding of $V$, also defined over $k$, into an affine space (onto a closed subset of an affine space if $V$ is affine) on which $G$ operates linearly.

This is an immediate consequence of the previous lemma.

LеммA 3. Let $\Gamma$ be a finitely generated commutative monoid, $\phi_{1}, \cdots, \phi_{,} a$ finite set of homomorphisms from $\Gamma$ into the additive group of integers $Z$. Then the submonoid of $\Gamma$ consisting of all $\gamma$ such that $\phi_{i}(\gamma) \geqq 0, i=1, \cdots, \nu$, is also finitely generated.

For the proof, it suffices to take $\nu=1$, so we consider a single $Z$-valued function $\phi$ on $\Gamma$. We may also take $\Gamma$ free, i.e.,

$$
\Gamma=\left\{\left(x_{1}, \cdots, x_{n}\right) \mid x_{1}, \cdots, x_{n} \in Z, x_{1}, \cdots, x_{n} \geqq 0\right\} .
$$

Write $\phi\left(x_{1}, \cdots, x_{n}\right)=\sum_{i=1}^{n} a_{i} x_{i}$, where $a_{1}, \cdots, a_{n} \in Z$, and let $\Gamma^{\prime}$ denote the submonoid consisting of all $(x) \in \Gamma$ such that $\phi(x) \geqq 0$. Excluding trivial cases, we may assume that no $a_{i}$ is zero, and that both positive and negative $a_{i}$ 's occur, say $a_{1}, \cdots, a_{r}>0, a_{r+1}, \cdots, a_{r+8}<0$, where $r, s>0, r+s=n$. Writing $a_{r+i}=-b_{i}, i=1, \cdots, s$, we have $b_{1}, \cdots, b_{s}>0$ and $\phi(x)$ $=a_{1} x_{1}+\cdots+a_{r} x_{r}-b_{1} x_{r+1}-\cdots-b_{s} x_{r+8}$. For each $\alpha=1, \cdots, r$ and 
$\beta=1, \cdots, s$, we define $\gamma_{\alpha \beta}=\left(\xi_{1}, \cdots, \xi_{r+8}\right) \in \Gamma$ by $\xi_{i}=\delta_{i \alpha} b_{\beta}, i=1, \cdots, r$ and $\xi_{r+i}=\delta_{i \beta} a_{\alpha}, i=1, \cdots, s$; clearly $\phi\left(\gamma_{\alpha \beta}\right)=0$, so $\gamma_{\alpha \beta} \in \Gamma^{\prime}$. If now $\left(x_{1}, \cdots, x_{r+\varepsilon}\right)$ is an arbitrary element of $\Gamma^{\prime}$ and at least one of $x_{r+1}, \cdots, x_{r+s}$ is very large, say $>M$ for some suitably large $M$, then at least one of $x_{1}, \cdots, x_{r}$ will be large, which implies that $(x)=\gamma_{\alpha \beta}+\left(x^{\prime}\right)$, for suitable $\alpha, \beta$ and a certain $\left(x^{\prime}\right) \in \Gamma^{\prime}$. That is, $\Gamma^{\prime}$ is generated by the various $\gamma_{\alpha \beta}$ 's together with those of its elements $(x)$ for which $x_{r+1}, \cdots, x_{r+s} \leqq M$. For each $\alpha=1, \cdots, r$ define $\gamma_{\alpha}=\left(\zeta_{1}, \cdots, \zeta_{r+s}\right)$ by $\zeta_{i}=\delta_{i \alpha}, i=1, \cdots, r+s ;$ then $\gamma_{1}, \cdots, \gamma_{r} \in \Gamma^{\prime}$, and moreover there exists a number $N$ such that if $(x) \in \Gamma^{\prime}$ and $x_{r+1}, \cdots, x_{r+s}$ $\leqq M$, while at least one of $x_{1}, \cdots, x_{r}$ is $>N$, then $(x)=\gamma_{\alpha}+\left(x^{\prime \prime}\right)$ for some $\alpha=1, \cdots, r$ and suitable $\left(x^{\prime \prime}\right) \in \Gamma^{\prime}$. Hence $\Gamma^{\prime}$ is generated by the various $\gamma_{\alpha \beta}$ 's and $\gamma_{\alpha}^{\prime}$ 's and by those $(x) \in \Gamma^{\prime}$ for which $x_{1}, \cdots, x_{r} \leqq N, x_{r+1}, \cdots, x_{r+\text {. }}$ $\leqq M$. Since these latter elements are in finite number, we are done.

In what follows, the translation of a function on a variety $V$ on which a group $G$ operates by an element $g \in G$ will be denoted $\lambda_{g}$; i.e., if $f \in \Omega(V)$ then $\lambda_{o} f(v)=f\left(g^{-1} v\right)$, for $v$ generic in $V$. The following result holds also, in an obvious sense, for algebraic sets that are reducible.

THEOREM 1. Let the torus $G$ operate regularly on the affine variety $V$, and suppose that all $G$-orbits on $V$ have the same dimension. Then $V / G$ exists and is also affine. If $W$ is a $G$-invariant subvariety of $V$, then we have a natural embedding $W / G \subset V / G$. If $V$ is a principal space for $G, V$ is actually a $G$-principal fiber bundle over $V / G$.

If $k$ is an algebraically closed field of definition for everything and $v$ a generic point of $V$ over $k$ then the isotropy subgroup of $v$, being a subgroup of the torus $G$, is defined over $k$, hence leaves each point of $V$ fixed. Dividing $G$ by this subgroup, we are reduced to the case where each orbit has dimension $\operatorname{dim} G$, i.e., each isotropy subgroup is finite. By Proposition 2 and the remark following, we may assume $G=G_{m}$. Indeed, letting $t$ be a multiplicative parameter on $G_{m}$, using Lemma 2 and recalling the diagonalizability of any representation of a torus, we may suppose $V$ to be a subvariety of the affine space $\Omega^{n}$ such that the coordinate functions $x_{1}, \cdots, x_{n}$ on $V$ transform by $G$ according to the rule $\lambda_{t}\left(x_{i}\right)=t^{v_{i} x_{i}}, i=1, \cdots, n$, where $\nu_{1}, \cdots, \nu_{n}$ are certain integers; the condition that all isotropy groups be finite is that for each $p \in V$ there exists $i=1, \cdots, n$ such that $\nu_{i} \neq 0$ and $x_{i}(p) \neq 0$. If $X_{1}, \cdots, X_{n}$ are indeterminates and we define the weight of a monomial $c X_{1}^{\alpha_{1}} \cdots X_{n}^{\alpha_{n}}$ (where $c \in \Omega, c \neq 0)$ to be $\alpha_{1} \nu_{1}+\cdots+\alpha_{n} \nu_{n}$, then any $F \in \Omega[X]$ can be written uniquely as $F=\sum_{i=-\infty}^{\infty} F_{i}$, where each nonzero $F_{i}$ is in $\Omega[X]$ and isobaric of weight $i$. If $F$ vanishes on $V$, so does each $F_{i}$, since $0=\lambda_{t} F\left(x_{1}, \cdots, x_{n}\right)$ $=F\left(t^{\nu_{1}} x_{1}, \cdots, t^{\nu} x_{n}\right)=\sum_{i} t^{i} F_{i}\left(x_{1}, \cdots, x_{n}\right)$, so the ideal of $V$ is generated by its isobaric elements and we have an induced grading on the ring $\Omega[V]=\Omega[x]$, an element of $\Omega[x]$ being isobaric of weight zero if and only if it is $G$-invari- 
ant. By Lemma 3, the multiplicative monoid of monic monomials in $X_{1}, \cdots, X_{n}$ of weight zero is finitely generated. Hence we may suppose that $\nu_{1}, \cdots, \nu_{r}=0, \nu_{r+1}, \cdots, \nu_{n} \neq 0$, and that the subring of $G$-invariant elements of $\Omega[V]$ is $\Omega\left[x_{1}, \cdots, x_{r}\right]$. The condition that all isotropy groups be finite is that the ideal generated by $x_{r+1}, \cdots, x_{n}$ be the unit ideal, implying in particular that for any $p \in V$ at least one of $x_{r+1}, \cdots, x_{n}$ of positive weight and at least one of negative weight do not vanish at $p$. Thus $\nu_{r+1}, \cdots, \nu_{n}$ include both positive and negative elements and, if we make the permissible assumption that $\nu_{r+1}, \cdots, \nu_{n}$ are relatively prime, $\Omega[V]$ contains isobaric elements of all integral weights. We are now in a position to prove that the map $\tau: p \rightarrow\left(x_{1}(p), \cdots, x_{r}(p)\right)$ gives us $V / G$ as an affine variety. First, any maximal ideal $\mathrm{m}$ of $\Omega\left[x_{1}, \cdots, x_{r}\right]$ extends to an ideal of $\Omega[V]$ that is not the unit ideal, for if we have a relation $\sum F_{i} m_{i}=1$, with each $F_{i} \in \Omega[V], m_{i} \in \mathfrak{m}$, the same relation would hold with each $F_{i}$ replaced by its isobaric component of weight zero, in which case $F_{i} \in \Omega\left[x_{1}, \cdots, x_{r}\right]$ so $1 \in \mathfrak{m}$ contrary to assumption. Thus the image $\tau V$ is a closed subset of $\Omega^{r} . \tau$ is clearly everywhere defined and constant on orbits. We need next to show that for any $p \in V$ the image $\tau p$ determines the orbit of $p$, and this we do as follows: Noting that a suitable power product of any two given elements of $\Omega[V]$ that are isobaric of positive and negative weights respectively will be in $\Omega\left[x_{1}, \cdots, x_{r}\right]$, we see that $\tau p$ determines which of $x_{1}(p), \cdots, x_{n}(p)$ are zero and which are nonzero. We can also find $y_{1}, y_{2} \in \Omega[V]$, power products of $x_{1}, \cdots, x_{n}$ that are not zero at $p$ and of weights respectively \pm g.c.d. $\left(\left\{\right.\right.$ all $\nu_{i}$ 's such that $\left.\left.x_{i}(p) \neq 0\right\}\right)$. Translation of $p$ by a suitable element of $G$ will then give $y_{1}(p)=1$, and since $y_{1} y_{2} \in \Omega\left[x_{1}, \cdots, x_{r}\right], y_{2}(p)$ will also be determined, and hence each $x_{i}(p)$, $i=1, \cdots, n$, will be determined; i.e. a point $p \in V$, normalized by $y_{1}(p)=1$, is determined by $\tau p$. Thus $\tau V$ is, at least set-theoretically, simply $V / G$. To complete the proof that' $\tau$ actually gives us a quotient space, let $p \in V$ and let $f \in \Omega(V)$ be $G$-invariant and defined at $p$. Writing $f=g / h$; with $g, h \in \Omega[V]$, $h(p) \neq 0$, and letting $g_{i}, h_{i}$ be the isobaric components of $g, h$ of weight $i$, we have $f=\lambda_{t} f=\sum_{i} t^{i} g_{i} / \sum_{i} t^{i} h_{i}$, so $h_{i} f=g_{i}$ for all $i . h_{i}(p) \neq 0$ for some $i$, and we can suppose this happens for $i=0$, for otherwise we can replace $g, h$ by their products with a suitable power of the previously used $y_{1}$ or $y_{2}$; then $f=g_{0} / h_{0}$, with $g_{0}, h_{0} \in \Omega\left[x_{1}, \cdots, x_{r}\right]$ and $h_{0}(p) \neq 0$, proving that $f$ is defined at $\tau p$. Thus we actually have a quotient variety. If $W$ is a $G$-invariant subvariety of $V$ then $\tau W=W / G$, since the map $\tau$ depends only on the integers $\nu_{1}, \cdots, \nu_{n}$, which are the same for $W$ as for $V$ (modulo the fact, which occasions no difficulty, that certain of the $\nu_{i}$ 's may not be well-defined for $W$, since possibly $x_{i}=0$ on $W$ ). To complete the proof, suppose that $V$ is a principal space for $G$. Then the map $\left(p,\left(\lambda_{t} x_{1}(p), \cdots, \lambda_{t} x_{n}(p)\right)\right) \rightarrow t$ is everywhere defined, so g.c.d. $\left(\left\{\right.\right.$ all $\nu_{i}$ 's such that $\left.\left.x_{i}(p) \neq 0\right\}\right)=1$ for all $p \in V$. Referring back to the proof that $\tau$ separates orbits, for any given $p \in V$ the functions $y_{1}, y_{2}$ 
chosen for $p$ will also be valid in a neighborhood of $p$. Setting $y_{1}=1$ then gives a cross section $V / G \rightarrow V$, valid in this neighborhood of $p$. Thus $V$ is indeed a principal fiber bundle.

Apropos of Theorem 1, we may make the following comments:

(1) If a torus $G$ operates regularly on a quasi-affine variety $V$, then $V$ is covered by its $G$-invariant affine open subsets. To show this, we may suppose that $V$ is embedded in an affine space $\Omega^{n}$ on which $G$ operates linearly. If $x_{1}, \cdots, x_{n}$ are the coordinate functions on $\Omega^{n}$ and $F$ is the complement of $V$ in its closure in $\Omega^{n}$, each translation by an element of $G$ maps $F$ into itself, hence each $\lambda_{v}$ maps the ideal of $F$ in $\Omega[x]$ into itself. By Lemma 1, we can find a set of generators $f_{1}, \cdots, f_{v} \in \Omega[x]$ for the ideal of $F$ such that each $\lambda_{0}$ induces a linear transformation on the vector space $\Omega f_{1}+\cdots+\Omega f_{v}$. Since any representation of a torus is diagonalizable, we may even assume that each $\lambda_{o}$ sends each $f_{i}$ into a multiple of itself. Then $V$ is covered by its $G$ invariant affine open subsets $\left\{V-\left(f_{i}=0\right)\right\}, i=1, \cdots, \nu$. However, all orbits on $V$ may have the same dimension and $V / G$ may still not exist: the remarks preceding Proposition 2 come into play, and the necessary conditions for piecing together the various local quotient varieties may not be satisfied. For an example, take $G=G_{m}, V=\Omega^{2}-(0,0)$, with the operation of $G$ on $V$ given by $(t,(x, y)) \rightarrow\left(t x, t^{-1} y\right)$.

(2) Under the conditions of Theorem 1, local (regular) cross sections need not exist if $V$ is not a principal space for $G$. If $V$ is a principal space there need not exist an everywhere regular cross section.

(3) If we relax the hypotheses of the theorem by supposing of the algebraic group $G$ merely that its component of the identity be a torus, then $V / G$ still exists and is still affine (but the other conclusions of the theorem do not generalize). For in view of the comment following Proposition 2, the original Theorem 1 gives an immediate reduction to the case where $G$ is finite. That $V / G$ exists and is affine when $V$ is affine and $G$ finite is a result of Serre [4, p. 57].

LEMMA. Let the unipotent algebraic group $G$ operate regularly on the quasiaffine variety $V$. Then any $G$-invariant rational function on $V$ is the quotient of two $G$-invariant rational functions on $V$ that are everywhere defined.

For suppose that $f_{1}, f_{2} \in \Omega[V]$, with $f_{1} / f_{2} G$-invariant. For all $c_{i} \in \Omega, g_{i} \in G$, we have $f_{1} / f_{2}=\sum c_{i} \lambda_{g_{i}} f_{1} / \sum c_{i} \lambda_{g_{i}} f_{2}$, provided that the last denominator is not zero. But the set of all $\lambda_{g_{i}} f_{2}^{\prime}$ 's span a finite dimensional vector space over $\Omega$ on which $G$ operates linearly. Since $G$ is unipotent, some nonzero element of this vector space is left fixed by each $g \in G$. If we choose $c_{i}, g_{i}$ such that $\sum c_{i} \lambda_{o_{i}} f_{2}$ is this nonzero fixed element, we are done.

The lemma can be sharpened slightly to add that if $G, V$ and the given $G$-invariant function on $V$ are all defined over $k$, then the two $G$-invariant 
elements of $\Omega[V]$ can actually be chosen in $k[V]$. For if these $G$-invariant elements are $\sum c_{i} \phi_{i}$ and $\sum c_{i} \psi_{i}$, with each $c_{i} \in \Omega$ and each $\phi_{i}, \psi_{i} \in k[V]$ and we take the various $c_{i}$ 's to be linearly independent over $k$, then linear disjointness and the fact that $\sum c_{i} \phi_{i} / \sum c_{i} \psi_{i} \in k(V)$ give that $\phi_{i} / \psi_{i}$ is independent of $i$ and each $\phi_{i}, \psi_{i}$ is $G$-invariant. This same rationality conclusion will of course hold in the more general situation where $G$ is no longer unipotent, but $G, V$ are such that the conclusion of the lemma still holds.

The following result is due to Kostant, at least in the classical case. The original proof was a complicated Lie algebra argument.

ThEOREM 2. Let the unipotent algebraic group $G$ operate regularly on the quasi-affine variety $V$. Then all orbits are closed.

We may assume that $G$ is connected and that $V$ is prehomogeneous with respect to $G$, i.e., that the generic orbit on $V$ is dense in $V$, and have to prove $V$ homogeneous. This is clear if $\operatorname{dim} G \leqq 1$, so assume $\operatorname{dim} G>1$ and use induction. Let $H \subset G$ be a connected normal algebraic subgroup of dimension 1 and consider the action of $H$ on $V$. By the lemma, there exist $x_{1}, \cdots, x_{n}$ $\in \Omega[V]$ such that the field of all $H$-invariant functions on $V$ is precisely $\Omega\left(x_{1}, \cdots, x_{n}\right)$. For $g \in G, h \in H, p \in V, i=1, \cdots, n$, we have $\lambda_{0} x_{i}(h p)$ $=x_{i}\left(g^{-1} h p\right)=x_{i}\left(g^{-1} h g g^{-1} p\right)=x_{i}\left(g^{-1} p\right)=\lambda_{g} x_{i}(p)$, so all translates of each $x_{i}$ are $H$-invariant. Thus we may suppose that the vector space $\Omega x_{1}+\cdots+\Omega x_{n}$ is invariant under each $\lambda_{g}, g \in G$. Define an everywhere defined rational map $\tau$ from $V$ onto a dense subset of an affine variety $V^{\prime}$ by $\tau(p)=\left(x_{1}(p), \cdots, x_{n}(p)\right)$. There is an induced operation of $G$ on $V^{\prime}$, linear and hence regular, such that $\tau(g p)=g(\tau p)$ for all $g \in G, p \in V$. But $H$ operates trivially on $V^{\prime}$, so $G / H$ operates regularly on $V^{\prime}$. Clearly $V^{\prime}$ is $G / H$-prehomogeneous, hence, by the induction assumption, homogeneous. To prove $V$ homogeneous it now suffices to show that, for any point $p \in V$, the points of $\tau^{-1}(\tau p)$ are translates of each other under $H$, and it is indeed enough to prove this when $p$ is generic for $V$ over $k, k$ being some field of definition for everything else. But then the points of $\tau^{-1}(\tau p)$ are precisely the $k(\tau p)$-specializations of $p$. Since $k(p)$ is a regular extension of $k(\tau p), \tau^{-1}(\tau p)$ is a variety, clearly prehomogeneous with respect to $H$. But $\operatorname{dim} H=1$, so $\tau^{-1}(\tau p)$ is homogeneous, which completes the proof.

Theorem 2 is not unreasonable in view of the known fact that any homogeneous space with respect to a connected unipotent group is biregularly equivalent to an affine space (proof by induction on dimension, using the existence of cross sections [3, Theorem 10] and the vanishing of coherent sheaf cohomology on affine varieties), but the point is that an affine space may be embedded in one of higher dimension as a nonclosed subset, e.g., map $\Omega^{2} \rightarrow \Omega^{3}$ by $(x, y) \rightarrow\left(x, x y, y+x y^{2}\right)$.

An immediate consequence of the theorem is that any conjugacy class of 
elements of a unipotent group is closed. More generally, on a connected solvable group, the conjugacy class of any element that is centralized by some maximal torus, e.g., any semisimple element, is closed.

The following lemma seems well known. The proof used here is from a manuscript of Borel.

Lemma. Let $G$ be a linear algebraic group defined over $k, H$ a $k$-closed subgroup. Then there exists a vector space $V$, defined over $k$, a faithful linear operation of $G$ on $V$, also defined over $k$, and a vector $v \in V$ that is rational over $k$, such that $H$ consists precisely of all elements of $G$ that map $v$ into a scalar multiple of itself.

In the algebra $\Omega[G]$ of everywhere defined rational functions on $G$ we may find a $G$-invariant finite dimensional subspace $S$ that contains a basis for the ideal of $H$. Since $G$ and $H$ are defined over $k^{p-\infty}$, we can find a basis $f_{1}, \cdots, f_{n}$ of $S$ such that each $f_{i} \in k^{p-\infty}[G]$ and $f_{1}, \cdots, f_{r}$ span the subspace of $S$ vanishing on $H$. Replacing $S$ by $S^{p^{*}}$ and $f_{1}, \cdots, f_{n}$ by their $p^{\prime \prime}$ th powers, if necessary, we obtain elements $f_{1}, \cdots, f_{n} \in k[G]$, linearly independent over $\Omega$, such that $S=\Omega f_{1}+\cdots+\Omega f_{n}$ is invariant under each $\lambda_{g}, g \in G$, such that $\Omega f_{1}+\cdots+\Omega f_{r}$ is precisely the subset of $S$ that vanishes on $H$, and such that whenever $g \in G$ and $f_{1}(g)=\cdots=f_{r}(g)=0$ we have $g \in H$. An element $g \in G$ is therefore in $H$ if and only if $\lambda_{0}$ maps $\Omega f_{1}+\cdots+\Omega f_{r}$ into itself. In the rational representation, defined over $k$, of $G$ as a group of linear transformations on $\Lambda^{r} S, H$ consists of precisely all elements of $G$ that map $f_{1} \Lambda \cdots \wedge f_{r}$ into a multiple of itself. If we let $V$ be the direct sum of $\Lambda^{r} S$ and a vector space on which $G$ operates faithfully, we are done.

THEOREM 3. Let $G$ be a connected linear algebraic groulp, $H$ an algebraic subgroup of $G$ whose component of the identity is either nilpotent, or possesses no nontrivial rational homomorphism into $G_{m}$. Then $G / H$ is a quasi-affine variety.

Assuming this for $H$ connected, the problem is to show that the quotient of a quasi-affine variety by a finite group of automorphisms exists and is also quasi-affine, which follows from Lemma 2 to Theorem 1 and the result of Serre quoted in comment (3) following Theorem 1 . Hence $H$ may be supposed connected. First suppose that $H$ possesses no characters. Then if $V$ and $v$ are as in the Lemma, we have $H v=v$ and the map $G \rightarrow V$ given by $g \rightarrow g v$ is a oneone rational map from $G / H$ onto a quasi-affine variety. $G / H$ is therefore the normalization of this latter variety in a larger field, hence itself quasi-affine. Finally, suppose $H$ nilpotent. Then $H=T \times U$, where $T$ is a torus and $U$ is unipotent. Since $G$ is an affine variety, so is $G / T$ (Theorem 1). Since $G / H$ $=(G / T) / U$, the lemma to Theorem 2 implies the existence of $f_{1}, \cdots, f_{n}$ $\in \Omega[G / T] \subset \Omega[G]$ such that $\Omega(G / H)=\Omega\left(f_{1}, \cdots, f_{n}\right)$. For each $g \in G, \lambda_{o}$ maps $\Omega[G] \cap \Omega(G / H)$ into itself, so we may suppose that the vector space $\Omega f_{1}+\cdots+\Omega f_{n}$ is invariant under all $\lambda_{g}$. $G$ operates linearly on this vector 
space, and we have an everywhere defined rational map from $G$ into the vector space given by $\gamma \rightarrow\left(f_{1}(\gamma), \cdots, f_{n}(\gamma)\right)$, this map commuting with left translation by elements of $G$. The image of $G$ is a homogeneous space for $G$, actually $G / H$ since $\Omega\left(f_{1}, \cdots, f_{n}\right)=\Omega(G / H)$. Therefore $G / H$ is quasi-affine.

The $G / H$ of Theorem 3 need not be affine. For example, if

$$
G=S L(2), \quad H=\left\{\left(\begin{array}{ll}
1 & x \\
0 & 1
\end{array}\right)\right\},
$$

then the map $G \rightarrow G / H$ is given by

$$
\left(\begin{array}{ll}
a & b \\
c & d
\end{array}\right) \rightarrow(a, c)
$$

and $G / H=\Omega^{2}-(0,0)$, which is not affine. On the other hand, Kostant has shown, by entirely different methods, that $G / H$ is affine whenever $H$ is reductive.

Corollary. If $G, H$ are as in the theorem and $U$ is a unipotent algebraic. subgroup of $G$, then the subset $U H$ of $G$ is closed.

For we may apply Theorem 2 to $U$ and $G / H$.

\section{REFERENCES}

1. C. Chevalley, Classification des groupes de Lie algebriques, Séminaire E.N.S., Paris, 1958.

2. - Fondements de la gémétrie algebrique, Paris, Secrétariat Mathématique, 1958.

3. M. Rosenlicht, Some basic theorems on algebraic groups, Amer. J. Math. vol. 78 (1956) pp. 401-443.

4. J.-P. Serre, Groupes algebriques et corps de classes, Paris, Hermann, 1959.

5. - Quelques propriettes des varietés abeliennes en caracteristique p, Amer. J. Math., vol. 80 (1958) pp. 715-739.

6. A. Weil, The field of definition of a variety, Amer. J. Math. vol. 78 (1956) pp. 509-524.

University of California,

Berkeley, California 